\title{
Having analyzed the RNA Structure of the Coronavirus COVID-19, the Methylation of its RNA Might Lead to the Neutralization of the Virus
}

\section{Krzysztof Nesterowicz ${ }^{1,2 *}$ and Maciej Świerczek ${ }^{2}$}

${ }^{1}$ National University of Public Service, Budapest, Hungary

${ }^{2}$ Faculty of Pharmacy, Medical College, Jagiellonian University, Krakow, Poland

*Corresponding Author: Krzysztof Nesterowicz, National University of Public Service, Budapest, Hungary.
Liposomes are nanocarriers comprised of lipid bilayers encapsulating an aqueous core. The ability of liposomes to encapsulate a wide variety of diagnostic and therapeutic agents has led to significant interest in utilizing liposomes as nanocarriers for theranostic applications [1]. Liposomes are spherical vesicles formed from lipid bilayers. Because of their unique structure, liposomes can entrap hydrophobic agents within the lipid bilayers and encapsulate hydrophilic agents inside the center aqueous compartment, which protects the agents from degradation. The inherent advantages of liposomes, such as high agent-loading efficiency, high stability in biological environments, controllable release kinetics, and biocompatibility, provide liposomes with better pharmacokinetics and biodistribution of theranostic agents than many other carriers in clinical studies [2]. A liposome can be obtained with the ACE2 receptor on the surface [3]. The SARS-CoV-2 virus binds to the liposome and fuses with it instead of attacking host cells. The liposome can contain: 1 . nothing - SARS-CoV-2 is sequestered inside and hence neutralized, 2. methylating agent, e.g. DMSO [4], 3. ribonuclease (RNAse) - neutralizes viral RNA, 4. antisense RNA or DNA strand.

\section{Bibliography}

1. Xing H., et al. "Recent Developments of Liposomes as Nanocarriers for Theranostic Applications". Theranostics 6.9 (2016): 1336-1352.

2. Deamer DW. "From "banghasomes" to liposomes: A memoir of Alec Bangham, 1921-2010”. FASEB Journal 24 (2010): 13081310 .
Received: May 14, 2021

Published: May 25, 2021

(C) All rights are reserved by Krzysztof

Nesterowicz and Maciej Świerczek.
3. Shereen MA., et al. "COVID-19 infection: Origin, transmission, and characteristics of human coronaviruses". Journal of Advanced Research 24 (2020): 91-98.

4. Iwatani M., et al. "Dimethyl sulfoxide has an impact on epigenetic profile in mouse embryoid body". Stem Cells 24.11 (2006): 2549-2556.

Volume 5 Issue 6 June 2021

(C) All rights are reserved by Krzysztof Nesterowicz and Maciej Świerczek. 